\title{
ANAYLSIS AND DETECTION OF COMMUNITY-ACQUIRED PNEUMONIA USING PSPNET WITH COMPLEX DAUBECHIES WAVELETS
}

\author{
Natzina Juanita S.R.F \\ Engineering School of Information Technology and Robotics, \\ Tomsk Polytechnic University, Lenin Avenue 30, Tomsk, Tomskaya Oblast, 634034, Russia \\ natzina_92@yahoo.com \\ Nadine Suzanne S.R.F \\ Engineering School of Information Technology and Robotics, \\ Tomsk Polytechnic University, Lenin Avenue 30, Tomsk, Tomskaya Oblast, 634034, Russia
}

\section{Shojaa Ayed Aljasar}

School of Nuclear Science and Engineering, Tomsk Polytechnic University, Lenin Avenue 30, Tomsk, Tomskaya Oblast, 634034, Russia

\section{Yubin Xu}

School of Nuclear Science and Engineering, Tomsk Polytechnic University, Lenin Avenue 30, Tomsk, Tomskaya Oblast, 634034, Russia

\section{Muhammad Saqib}

Research School of Chemistry and Applied Biomedical Sciences,

Tomsk Polytechnic University, Lenin Ave 30, Tomsk, Tomskaya Oblast, 634034, Russia

\begin{abstract}
Pneumonia is a lethal health ailment, often occurring in middle-aged and elderly patients. This malady is generally identified from critical common cold symptoms. In most test cases, multi imaging techniques are used rather than a single imaging technique to detect pneumonia. This is because varied multiple imaging techniques provide different values for easy analysis and identification of the disease. It is commonly known that pneumonia can be acquired in many ways, the most common being Community Acquired Pneumonia (CAP). The main aim of this research is to analyze and identify the spread of CAP as it occurs mainly in adults above 50 years of age. This is achieved by passing $C T$ images of infected lungs into Pyramid Scene Parsing Network (PSPNet) which is combined with pyramid pooling. This combination trains images to produce many output image subsets based on the number of abnormalities in the image. Complex Daubechies wavelets identify intricate details of the CAP affected areas in infected lungs to produce accurate results as required.
\end{abstract}

Keywords: Complex Daubechies Wavelets; Community-Acquired Pneumonia (CAP); Image Analysis; High Resolution Computer Tomographic Images (HRCT); Pyramid Scene Parsing Network (PSPNet), Pyramid Pooling.

\section{Introduction}

\subsection{Pneumonia - Types of Severity}

Pneumonia [1] is a chronic disease that is identified by the amount of fluid in the lung cavity caused by the growth of bacteria or fungi on both sides of the lungs. The most common symptoms of this disease are respiratory disorder, fever and cough. However, the seriousness of the disease is determined by the type and duration of the microbe causing it, and the overall health and age of the patient (infants below 5 years and elderly over 65 years). However, extra attention should be provided to detect pneumonia [1] in elderly patients (above 50 years of age), as symptoms are exhibited mildly, and the severity of the ailment increases even before it is accurately detected.

The type of pneumonia in the affected patient depends on whether the disease was acquired from within the hospital premises (commonly known as hospital-acquired pneumonia) or if it was acquired from outside the hospital premises (commonly known CAP) [2,3]. CAP is a more commonly acquired disease and hence the research was carried out for this type for detection. 
CT scans were collected from local health institution and it consisted of 328 patients who were diagnosed with CAP $[2,3]$. The age group of the patients and the severity of the disease are shown below:

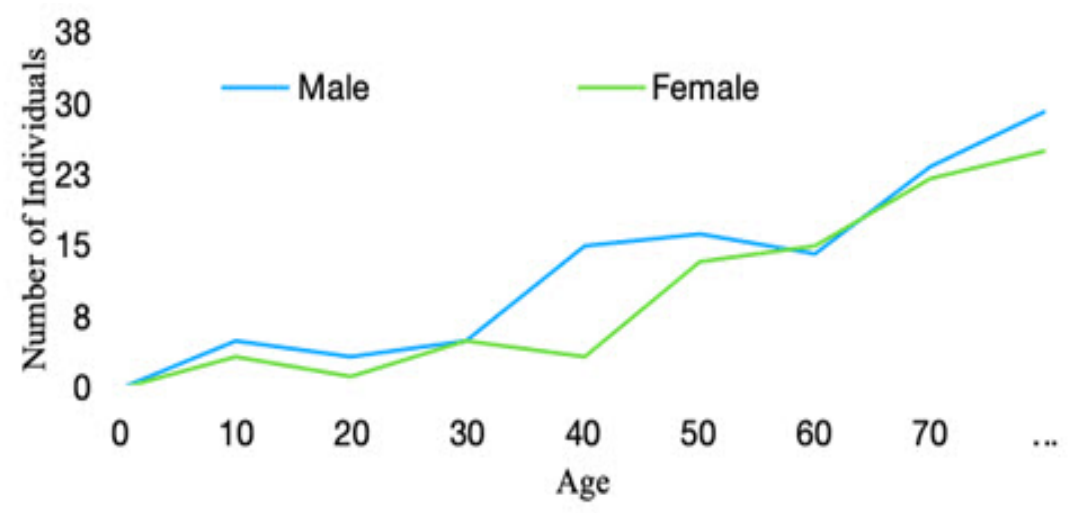

Fig. 1. Patients diagnosed with CAP according to their age. X-Axis represents the age of the affected patients with CAP and the Y-Axis represents the probability of individuals affected with CAP

In fig 1 , the data displayed depicts people above 50 years of age being most affected with pneumonia when compared to the number of patients of a younger age.

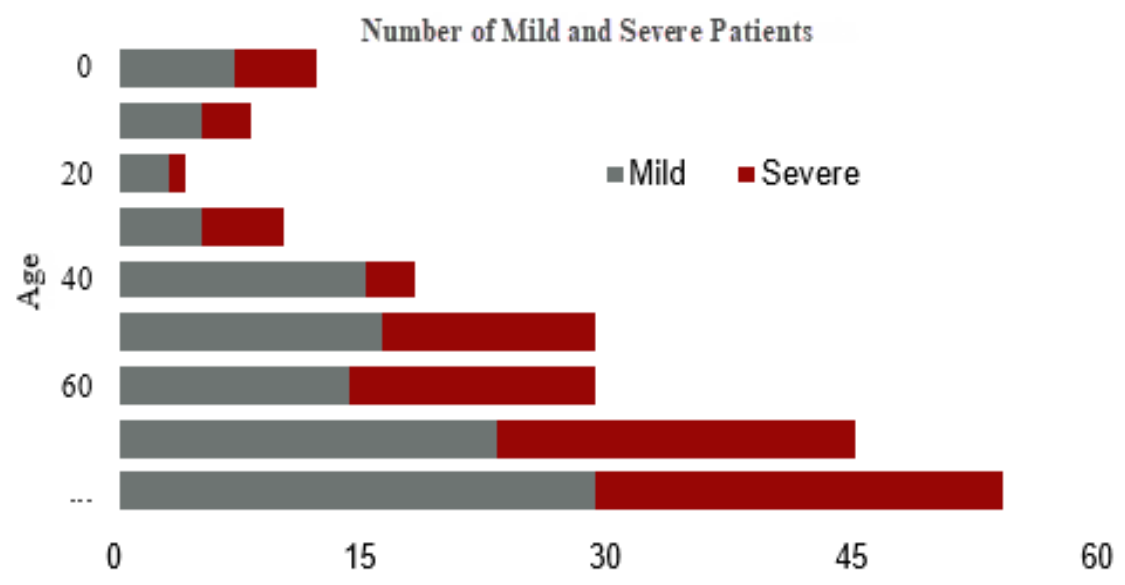

Fig. 2. A Graphical Representation of severity of CAP according to their age. X-Axis represents an approximate of patients affected by CAP with a difference in the mild and severe cases and the Y-Axis represents the age at which a person is most likely to be affected.

Fig 2 depicts how the severity of patients affected with CAP increases with age. Due to the above recorded facts, further research is performed to detect and analyse of CAP in elderly affected patients.

\subsection{Detection of CAP using various image analysis techniques}

Through the years, various imaging techniques [4] have been used for the detection of CAP in lungs. Sometimes, image outputs provide only false positives and false negatives during analysis. The imaging techniques [5,6] used mostly today for detecting CAP in lungs are as follows:

- X-Ray. Chest X-Rays [7] are used for image analysis as they are easy to operate and cost less. They can be used for detecting CAP in lungs as image outputs provide a lot of related information. However, due to tissues overlapping each other detection of affected areas in the lung is difficult. There is usually a small amount of missed detection even in the presence of Computer Aided Devices (CAD) systems[8]. The complexity of lung images obtained from X Rays is so high that the automatic detection of diseases from them provide minimal results than expected.

- Computer Tomography (CT). A series of X-Ray images form a CT image. Since CT images provide more detailed information than X-Rays, they are better used for analysis of CAP in lungs. During recent findings, CT scan [8] helped effective re-labelling of images which improved analysis from $8 \%$ to $18 \%$. 
The calculation of CAP diagnosis [9] using various analysis techniques have been calculated and its values are presented in table 1 below.

Table 1. Comparison of accuracy CT and X-Ray image analysis techniques

\begin{tabular}{|l|l|l|}
\hline Performance & X-Ray & CT Scan \\
\hline Irradiation (+/-) & + & + \\
\hline Sensitivity & $52 \%$ & $83 \%$ \\
\hline Specificity & $41 \%$ & $76 \%$ \\
\hline Lung pathology & 0.08 & 0.49 \\
\hline
\end{tabular}

In Table 1, it is seen that the accuracy of using CT image techniques in terms of its irradiation and cost have proven to be a better system for diagnostic purposes. The CT scan technique depicts a betterment of about $30 \%$ of sensitivity [10] and specificity [11] when compared to the X-Ray technique. According to Prendki et al [12], false negatives and false positives calculated for CT images containing CAP is calculated as 0.27 and 0.36 . The most successful detection of CAP was found in patients with an intermediate CAP probability.

The proposed network architecture is designed to eliminate existing problems in identifying CAP. It also improves the values of sensitivity and specificity, which thereby increases the accuracy of detection.

\section{Methods and Technologies}

The proposed method includes the following four phases:

- The affected lung CT images are analysed and grouped according to their similarities into subsets.

- These subsets are passed into PSPNet [13] and failed subset occurrences are identified and classified as inconspicuous classes

- All the subsets are then combined through pyramid pooling [13] to provide a combined abnormality model with lung border.

- From this abnormality model, complex Daubechies wavelets are used to identify CAP affected areas.

The datasets used in the system are of high-resolution computer tomography (HRCT) images of patients suffering from CAP. The images are taken for affected patients above 50 years of age.

\subsection{Identifying Data Similarities}

The ideal way to identify the similarities in images is to extract their features and compute them. To achieve this, a multi scale convolutional neural network $(\mathrm{CNN})$ that uses deep ranking [14] is used.
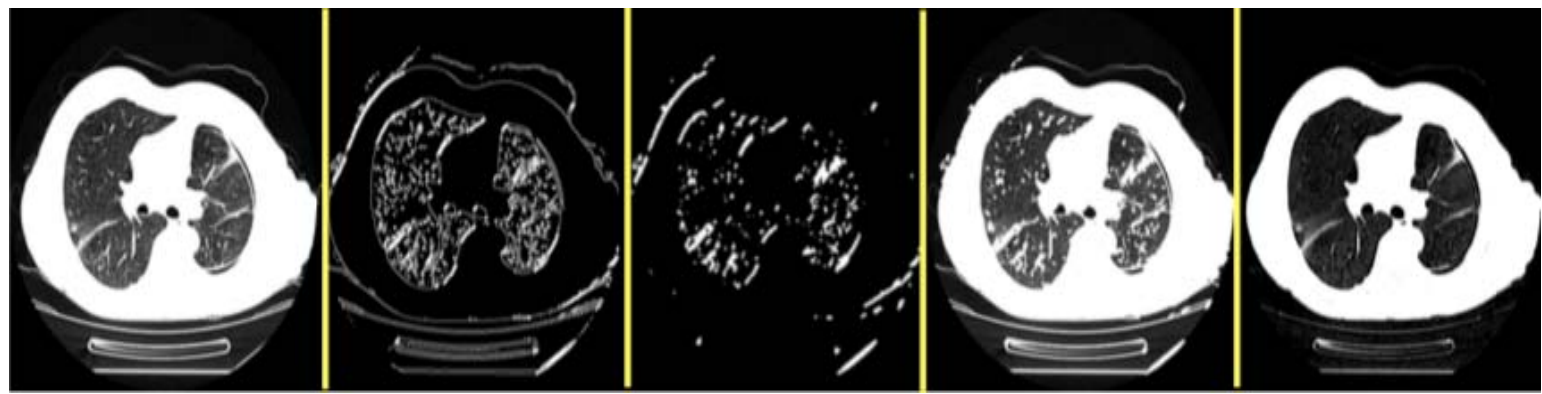

Fig. 3. Computer Tomographic Scans with different textures and variations with various pathological condition

During this process, images are categorized by calculating the square of the Euclidean metric into three subsets, namely query, positive and negative. This Euclidean metric is calculated by the formula.

$$
\mathrm{D}(\mathrm{f}(\mathrm{pi}), \mathrm{f}(\mathrm{pi}+))<\mathrm{D}(\mathrm{f}(\mathrm{pi}), \mathrm{f}(\text { pi-) }) \text { such that } \mathrm{r}(\mathrm{pi}, \mathrm{pi}+)>\mathrm{r}(\text { pi,pi-) }
$$

where, the embedding function [15] for the Euclidean metric is represented by ' $\mathrm{f}$ ' to map the image to a vector, the pi, pi+ and pi - represents the query, positive, and negative images respectively and ' $r$ ' represents the distance of similarity [16] between any two images.

In this way, the image is classified into 3 subsets. While repeating the process, the embedding metric [16] determines similar properties in every image and the hinge loss for each image is calculated as follows:

$$
\mathrm{I}(\text { pi,pi+, pi- })=\max \{0, \mathrm{~g}+\mathrm{D}(\mathrm{f}(\mathrm{pi}), \mathrm{f}(\mathrm{pi}+))-\mathrm{D}(\mathrm{f}(\mathrm{pi}), \mathrm{f}(\text { pi- }))\}
$$


where, $\mathrm{D}$ is the Euclidean distance and $\mathrm{g}$ is the parameter that regularises the gap caused by the distance.

\subsection{Dealing with Unidentified image data}

Once the CT images have been grouped according to their similarities, the textures of these images [17] are almost similar. These image subsets then pass into a Pyramid Scene Parsing Network (PSPNet) $[18,19]$, where the images are scaled into different sizes [20]. During this method, complex scene parsing takes place, where the unidentified abnormalities from the multiscale CNN [21] are identified and grouped into inconspicuous classes [22]. In order to avoid false data accumulation, context relationship [23,24] is kept between images. These inconspicuous classes are also saved in separate subsets. The analysis of this data can be verified, and cross checked with the details of the affected patients suffering from CAP that is input into the system.

\subsection{Image Output via PSPNet}

The complex condition of the HRCT images uses pyramid pooling method to cater to the variation of the datasets [25]. This method is used to fuse the features extracted at four different pyramid scales. After every layer of the pyramid, a $1 * 1$ convolutional layer is used to maintain the feature weight. The dimension is thus $1 / \mathrm{N}$ where $\mathrm{N}$ is the pyramid. The technique of bilinear interpolation [26] is done to get the same size of the feature $[27,28]$ that was originally input into the system. The image output from the pyramid pooling module [29] displays with all abnormalities and the image border.

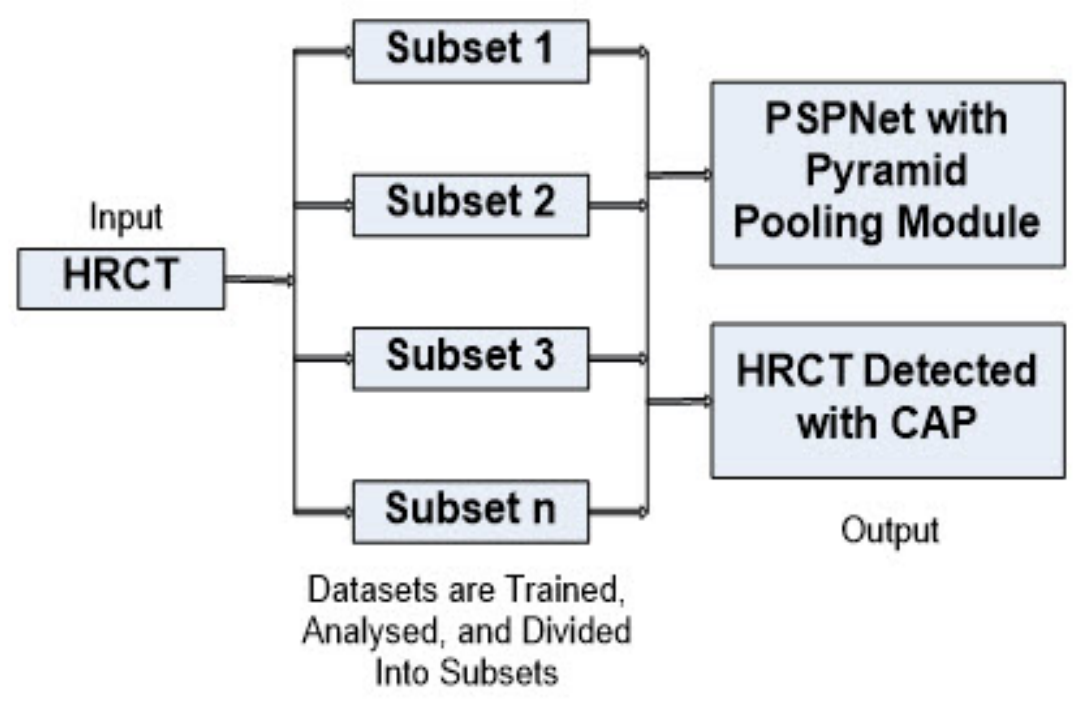

Fig. 4. The network architecture of the overall working process to analyse and detect CAP

\subsection{Complex Daubechies Wavelets used for scaling pixel coefficients}

The final process of identifying CAP in affected patients involves manipulating complex Daubechies [30] Wavelet properties in pyramid pooling, once the output image with abnormalities is obtained. These wavelets scale the coefficients to identify CAP. In order to do so, consider the real and imaginary scaling functions [31], which are defined as follows

$$
\begin{aligned}
& \mathrm{A}(\mathrm{x}, \mathrm{y})=\mathrm{h}(\mathrm{x}) \mathrm{h}(\mathrm{y})-\mathrm{g}(\mathrm{x}) \mathrm{g}(\mathrm{y}) \simeq \mathrm{K}(\mathrm{x}, \mathrm{y}) \\
& \mathrm{B}(\mathrm{x}, \mathrm{y})=\mathrm{h}(\mathrm{x}) \mathrm{g}(\mathrm{y})+\mathrm{g}(\mathrm{x}) \mathrm{h}(\mathrm{y}) \simeq \alpha \Delta \mathrm{K}(\mathrm{x}, \mathrm{y})
\end{aligned}
$$

where, $A(x, y)$ and $\beta(x, y)$ represent the real and imaginary parts of the image, $K(x, y)$ represents the smoothing kernel, $\mathrm{h}(\mathrm{x}) \mathrm{h}(\mathrm{y}), \mathrm{g}(\mathrm{x}) \mathrm{g}(\mathrm{y}), \mathrm{h}(\mathrm{x}) \mathrm{g}(\mathrm{y})$ and $\mathrm{g}(\mathrm{x}) \mathrm{h}(\mathrm{y})$ represent Fourier's calculations to compute the scaling functions and $\alpha \Delta \mathrm{K}(\mathrm{x}, \mathrm{y})$ represents the Laplacian of Gaussian (LOG) function.

The real part is equivalent to the smoothing kernel while the imaginary part is proportional to the LOG function, which makes the latter a "Marr" wavelet of the former. These functions provide an elementary operation on the wavelet coefficients of the pixels identified from scaling. The function denotes the convolution of the original area where the Laplacian [32] of the image can be multi-scaled or smoothened. Since the image is divided into the real and imaginary parts, only the real part of the image is kept while the imaginary part and pixels representing them are removed. The ability of the real part to interpolate its properties is then made use of, and the scaling coefficient is calculated using the equation:

$$
\mathrm{P}_{\mathrm{R}}\left(\Sigma_{\mathrm{a}, \mathrm{b}}\left(\mathrm{x}^{\mathrm{j} \max }+\mathrm{iy}^{\mathrm{j} \max }\right) \varphi_{\mathrm{j} \max , \mathrm{a}, \mathrm{b}}(\mathrm{m}, \mathrm{n})\right)=\Sigma_{\mathrm{a}, \mathrm{b}} \mathrm{x}^{\mathrm{j} \max } \varphi_{\text {jmax }, \mathrm{a}, \mathrm{b}}(\mathrm{m}, \mathrm{n}) \text { where } \mathrm{x} \longrightarrow \mathrm{a}, \mathrm{b}
$$


where, for a point in space $\mathrm{f}(\mathrm{x}, \mathrm{y})$, jmax represents the scaling coefficients, and $\mathrm{x}_{\mathrm{a}, \mathrm{b}}$ and $\mathrm{y}_{\mathrm{a}, \mathrm{b}}$ represents the real and imaginary parts of the scaling function, and $\mathrm{P}_{\mathrm{R}}$ is the orthogonal projector in a specified space. The operator to calculate the smoothing kernel [33] only for the real part of the projector is calculated as follows:

$$
\mathrm{S}: \mathrm{R}_{\mathrm{jmax}} \longrightarrow \mathrm{R}_{\mathrm{jmax}+1}
$$

where $S$ is the output image for the field points $R_{j \max }$ and $R_{j m a x+1}$.

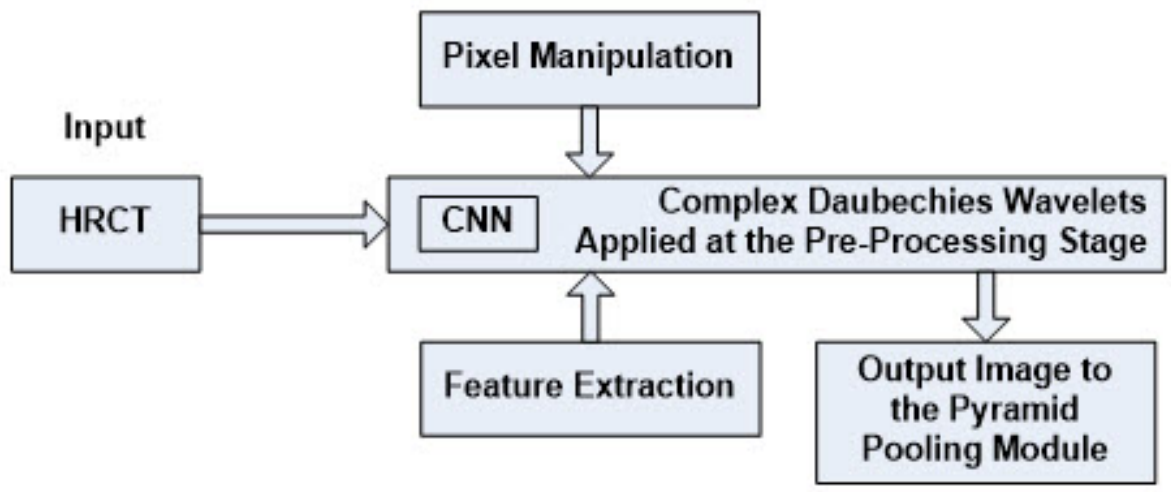

Fig. 5. The HRCT image trained in the CNN with Complex Daubechies Wavelets

The output image will be twice the size of the input image and will display without obsolete components of frequencies distorting the image. The real values which calculate to zero give the edge of the lung CT image [34] and these edges and should be ignored. The remaining real pixel values are calculated and projected. These correspond to CAP affected areas, which is the required output.

\section{Experimental Analysis}

The output analysis from the program for different patients with different severity of CAP are depicted in the output images. The performance of the model is evaluated statistically by $\mathrm{x} 2$ tests and Kruskal-Wallis tests. The Kruskal-Wallis tests the similarity property of the images in the subsets and $94 \%$ was found to be similar to each other. Following this, a comparison between continuous and dichotomous variables is done for a clear output analysis. The sensitivity and the specificity for the CAP images are also calculated.

The deep learning algorithm is trained for the execution of results and the time taken for this purpose is approximately 5.32 seconds. The process is executed using an 8GB GPU processor. The collected input datasets consist of 328 CT scans, of 247 CAP affected patients, aged about 50 and above. The test cases are performed keeping groups of 4 test samples of about $70 \mathrm{CT}$ images with a confidence interval of $95 \%$ in each sample. The sensitivity and specificity percentages of the samples are calculated to identify false positives and false negatives. The resulted values of each test case sample set for detecting CAP can be seen in table 2 below, where, sensitivity displays approximately 90-97 percent and specificity approximately 92-99 percent of positive results. Also, the tested image sets recoded an AUC (area under receiver operating characteristic curve) of approximately 0.954 .

Table 2. Statistical Analysis of CAP Affected Test Cases

\begin{tabular}{|l|l|l|l|l|}
\hline CAP Images & Sensitivity \% & Specificity \% & AUC & P-value \\
\hline Test Case 1 & 67 of $70(95.7)$ & 66 of $70(96)$ & 0.94 & $<0.001$ \\
& {$[84,92]$} & {$[82,94]$} & $0.93,0.95]$ & \\
\hline Test Case 2 & 63 of $70(89.96)$ & $\begin{array}{l}65 \text { of } 70(92) \\
{[88,94]}\end{array}$ & $\begin{array}{l}0.95 \\
{[0.92,0.97]}\end{array}$ & $<0.001$ \\
& {$[85,91]$} & 61 of $70(96)$ & 0.95 & $<0.001$ \\
\hline Test Case 3 & 65 out of $70(90)$ & {$[92,99]$} & $0.90,0.99]$ & \\
& {$[87,90]$} & 76 of $78(99)$ & 0.98 & $<0.001$ \\
\hline Test Case 4 & 76 out of $78(97)$ & {$[84,99]$} & {$[0.97,0.99]$} & \\
\hline & {$[95,97]$} & & & \\
\hline
\end{tabular}


The graph in fig 6, 7, 8 and 9 display as receiver operating characteristic (ROC) curve. The $\mathrm{x}$ and y values in each graph are plotted for statistical analysis of sensitivity and specificity. The AUC for each graph is calculated using DeLong's method to compare and analyze the working performance of the model.

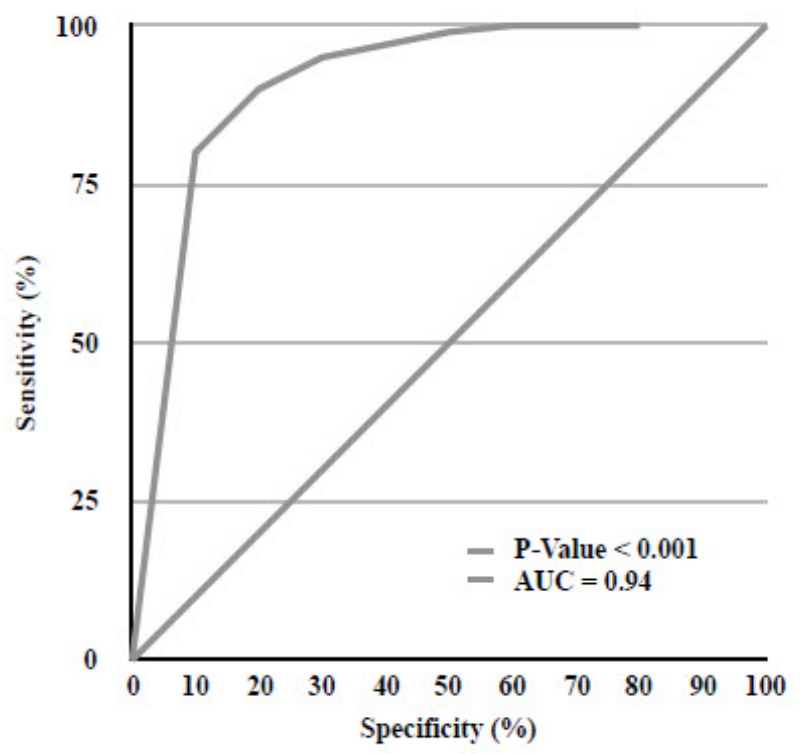

Fig. 6. ROC Curve for Test Case 1

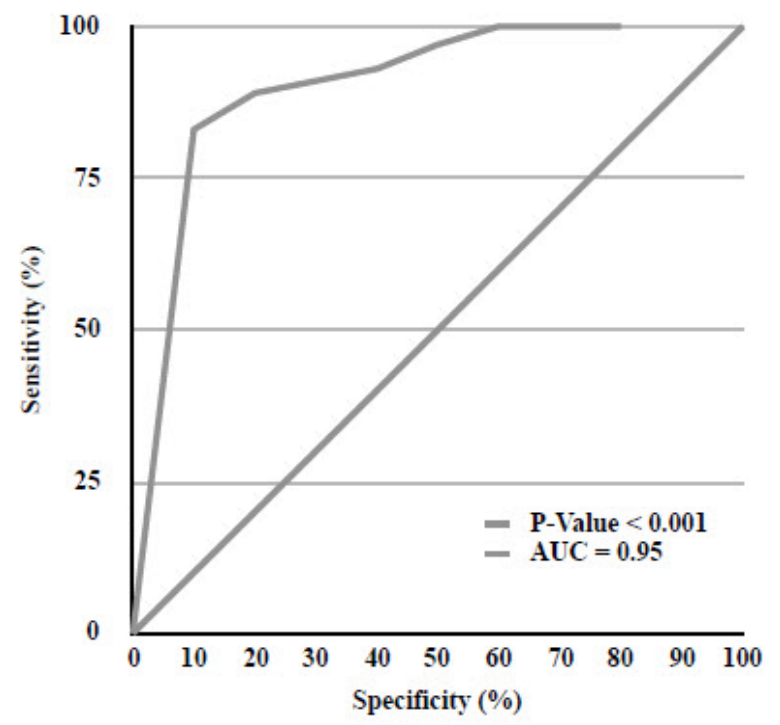

Fig. 7. ROC Curve for Test Case 2 


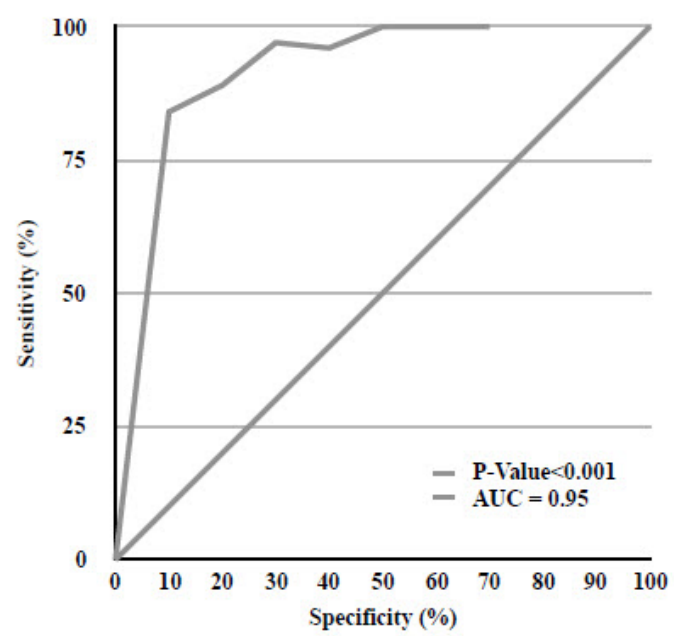

Fig. 8. ROC Curve for Test Case 3

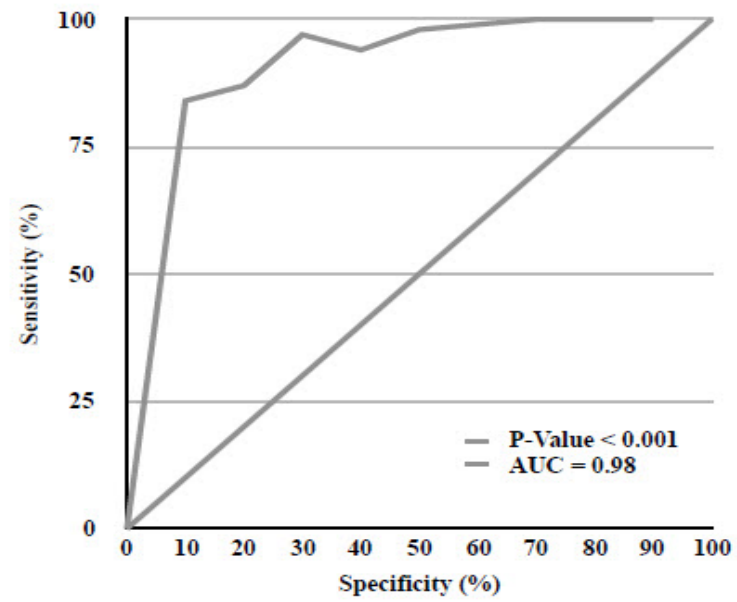

Fig. 9. ROC Curve for Test Case 4

From the above analysis, the calculated P-Value was identified to be 0.001 thus inferring the superior performance of the working model.

Fig 10 below, depicts varied image slices of patient 1 aged 87 years with an infected left lung. In fig 10,11 and 12, the visible green region in the image represents the CAP infected area and the red regions represent the nodules. In slice a, the severity of CAP is minimal. Even the nodules within the affected area are detected and displayed accurately. Slice b displays CAP at a minimal level where nodules are not visible. In slice c, the severity of CAP is huge. Hence, the CAP detection is visible only for the border.

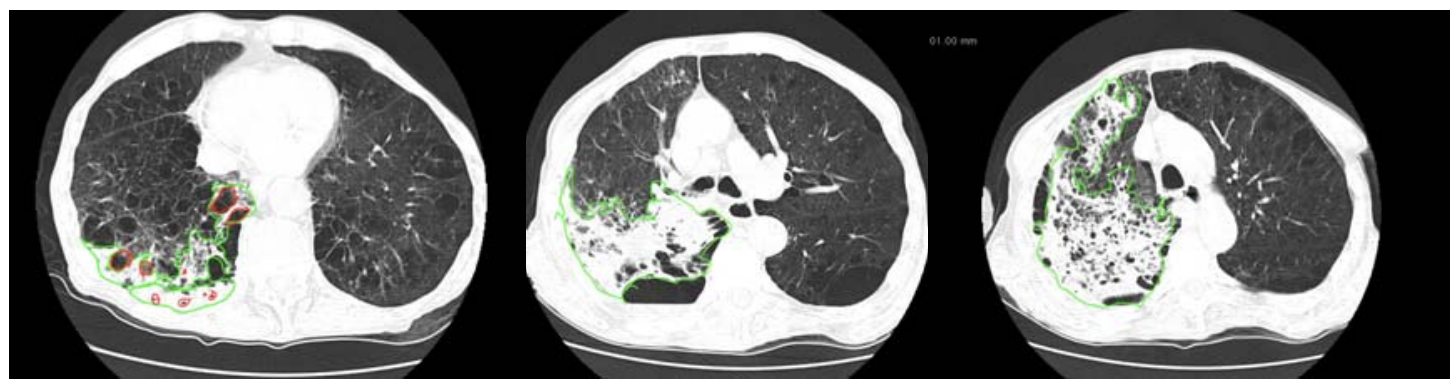

Fig. 10. Detected CAP in slice a, b and c (left to right) of patient 1 of 87 
Fig 11 below, depicts varied image slices of patient 2 aged 71 years and the output is determined for 3 slices with different levels of severity. The severity of CAP is more in patient 2, as both lungs are infected. Nodules are visible in slice 2. Sometimes the detected nodules lead to false negatives as they may not be actual nodules but of some other pathological conditions. Slice c of patient 2 displays the border area severely infected with CAP.

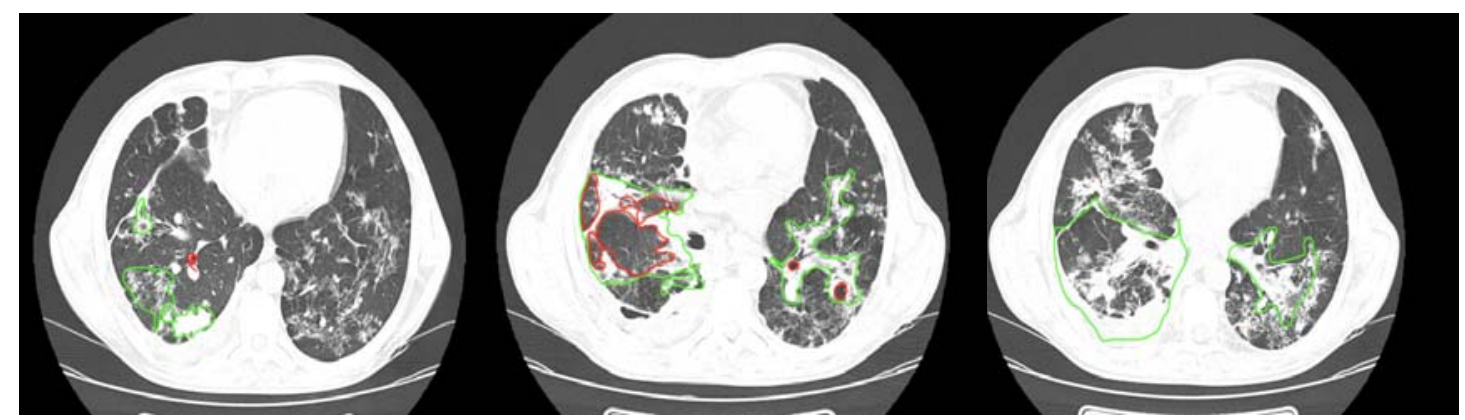

Fig. 11. Detected CAP in slice $a, b$ and $c$ (left to right) of patient 2 of 71 years

Fig 12 below, depicts varied image slices of patient 3 aged 53 years. Image slices of patient 3 has the most accurate output of all the detected CAP outputs. CAP deteriorates in the image showing the disappearance of CAP from the lungs. This shows a healthy improvement of the patient.

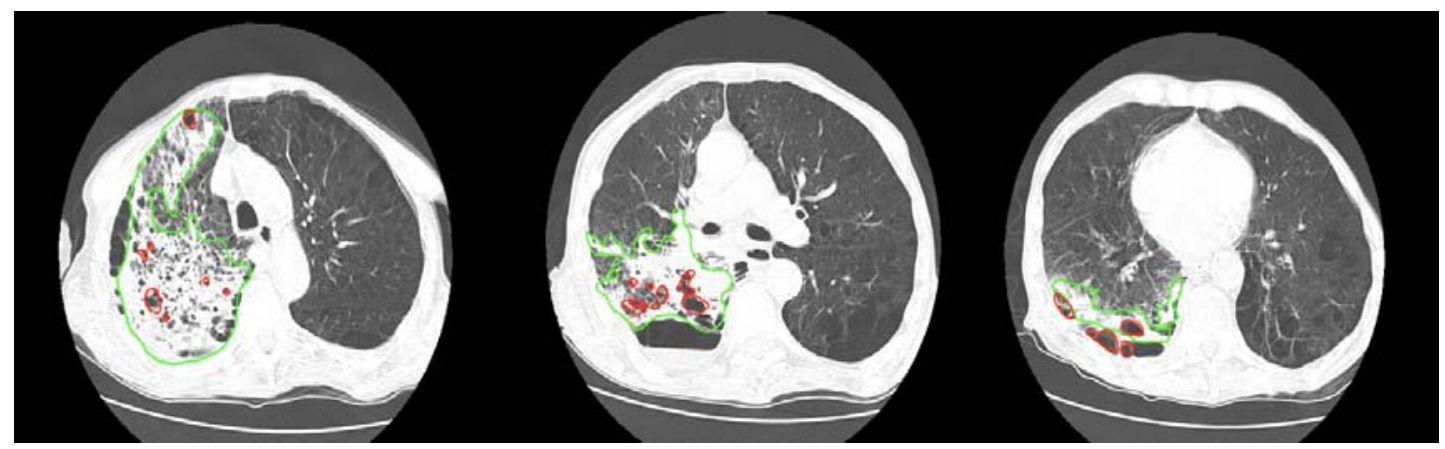

Fig. 12. Detected CAP in slice $a, b$ and c (left to right) of patient 3 of 53 years

Similarly, CAP was detected in CT images for around 247 patients of varied ages above 50.

Image Loss Analysis. As the image passes through PSP Net, there is a little output loss in the total number of output pixels. The auxiliary loss that the pixel output experiences is 0.03 . According to Hengshuang Zhao et al auxiliary loss should be 0.4 to toggle maximum output. But the loss can be decreased by enhancing the image output from the CNN with Daubechies wavelets, thus, contributing to the clear picture resolution obtained as an output.

\section{Conclusion}

The method of combining the PSPNet with the pyramid pooling module aids radiologists to accurately identifying Community-Acquired Pneumonia (CAP) in the early stages in affected patients. By a CNN, the HRCT images are better trained, analysed and grouped according to their pathologies into various subsets. The output images are parsed into the PSPNet with the pyramid pooling module to scale, identify abnormalities, upsample and resize images to produce the final output. The complex Daubechies Wavelets are applied to the image to identify the intricate detailing. By performing these methods, the output images display diseased regions containing CAP. Thus, the test case results are achieved with a certain amount of accuracy and efficiency. 


\section{References}

[1] Gharib, A.M., Stern, E.J. (2001). Radiology of pneumonia. Med. Clin. North Am.;85(6):1461-91.

[2] Bartlett, J.G. (2011). Diagnostic tests for agents of community-acquired pneumonia. Clin. Infect. Dis., 52 Suppl 4:S296-304.

[3] Hage, C.A., Knox, K.S., Wheat, L.J. (2012) Endemic mycoses: overlooked causes of community acquired pneumonia. Respir Med.106(6):769-76.

[4] Claesson, T. (2001). A Medical Imaging Demonstrator of Computed Tomography and Bone Mineral Densitometry, Master Thesis, Department of Physics, Royal Institute of Technology, Stockholm, Sweden.

[5] Roobottom, C. A., Mitchell, G., Hughes, G. M. (2010) Radiation-Reduction Strategies in Cardiac Computed Tomographic Angiography, Clinical Radiology, vol. 65, no. 11, pp. 859-867.

[6] Mehmood, I., Ejaz, N., Sajjad, M., Baik, S.W. (2013) Prioritization of Brain MRI Volumes Using Medical Image Perception Model A Tumor Region Segmentation, Computers in Biology and Medicine, vol. 43, no. 10, pp. 1471-1483.

[7] Xu, J., Tsui, B.M.W. (2014) Quantifying the Importance of the Statistical Assumption in Statistical X-ray CT Image Reconstruction, IEEE Transactions on Medical Imaging, vol. 33, no. 1, pp. 61 - 73.

[8] Hiriyannaiah, H. P., Cupertino, C. A. (1997). X-ray Computed Tomography for Medical Imaging, IEEE Signal Processing Magazine, vol. 14 , no. 2 , pp. $42-59$.

[9] Jaiswal, A.K., Prayag, T., Deepak, G., Ashish, Khanna., Joel, R. (2019). Identifying Pneumonia in Chest X-Rays: A Deep Learning Approach. Measurement. 145. 10.1016/2019.05.076.

[10] Li, W., Zhu, X., Li, J., Peng, C., Chen, N., Qi, Z., Yang, Q., Gao, Y., Zhao, Y., Sun, K., \& Li, K. (2014). Comparison of the sensitivity and specificity of 5 image sets of dual-energy computed tomography for detecting first-pass myocardial perfusion defects compared with positron emission tomography. Medicine, 93(28), e329.

[11] Trevethan R. (2017). Sensitivity, Specificity, and Predictive Values: Foundations, Pliabilities, and Pitfalls in Research and Practice. Frontiers in public health, 5, 307. https://doi.org/10.3389/fpubh.2017.00307.

[12] Prendki, V., Max, S., Benedikt, H., Nicolas, G., François, H., J. (2018). Low-dose CT for the diagnosis of pneumonia in elderly patients: a prospective, interventional cohort study, European Respiratory Journal, 1702375; DOI: 10.1183/13993003.02375-2017.

[13] Han, X.; Zhong, Y.; Cao, L.; Zhang, L. (2017). Pre-Trained AlexNet Architecture with Pyramid Pooling and Supervision for High Spatial Resolution Remote Sensing Image Scene Classification. Remote Sens. 9, 848

[14] Sharma, R.,Vishvakarma,A. (2019). Retrieving Similar E-Commerce Images Using Deep Learning, pp.1901-03546.

[15] Linardi, M., Zhu, Y., Palpanas, T., Keogh, E., J. (2018). Matrix Profile X: VALMOD - Scalable Discovery of Variable-Length Motifs in Data Series. In Proceedings of the International Conference on Management of Data (SIGMOD). pp.1053-1066.

[16] Prasad, S., Shehata, M. (2017). Image matching using SIFT, SURF, BRIEF and ORB: performance comparison for distorted images." arXiv preprint arXiv: 1710.02726

[17] Simon, W., Lucio, C., Mario, S., Nicola, S. (1998). A Deep Learning Algorithm for Classifying Fibrotic Lung Disease on High Resolution Computed Tomography. European Respiratory Journal, 52: OA262; DOI: 10.1183/13993003.congress-2018.OA262.

[18] Huiying, L., Anyang, S., Chang, L., Yunqiang, W., Shengbo, C. (2019). Bisupervised network with pyramid pooling module for land cover classification of satellite remote sensing imagery," J. Appl. Rem. Sens. 13(4) 048502.

[19] Kaiming, H., Xiangyu, Z., Shaoqing, R., and Jian, S. (2014). Spatial pyramid pooling in deep convolutional networks for visual recognition. In European conference on computer vision, pp.346-361.

[20] Ziwei, L., Xiaoxiao, L., Ping L., Chen-Change, L., Xiaoou, T. (2015). Semantic image segmentation via deep parsing network. In Computer Vision (ICCV), 2015 IEEE International Conference on, pp.1377-1385.

[21] Walsh, S.L.F., Calandriello, L., Silva, M., Sverzellati, N. (2018). Deep learning for classifying fibrotic lung disease on high-resolution computed tomography: a case-cohort study. Lancet Respir Med.

[22] McBee, M.P., Awan, O.A., Colucci, A.T. (2018). Deep learning in radiology. Acad Radiol. doi: 10.1016/j.acra.2018.02.018.

[23] Samek, W., Wiegand, T., Muller, K.R. (2018) Explainable artificial intelligence: Understanding, visualizing and interpreting deep learning models. ITU Journal: ICT Discoveries - Special Issue 1 - The Impact of Artificial Intelligence (AI) on Communication Networks and Ser- vices 1(1), pp. 39-48.

[24] Zhao, Q., Feng, W., Wan, L., Zhang, J. (2015). SPHORB: A Fast and Robust Binary Feature on the Sphere, Int. J. Comput. Vision 113 (2), pp.143-159.

[25] Fan, M., Huang, R., Feng, W., Sun, J. (2017). Image blur classification and blur usefulness assessment, in: Proceedings of the 2012 IEEE International Conference on Multimedia \& Expo Workshops (ICMEW).

[26] Fadnavis, S. (2014). Image Interpolation Techniques in Digital Image Processing: An Overview. International Journal of Engineering Research and Application. 4. 2248-962270.

[27] Lavanya, M., MuthuKannan, P. (2017). Lung Lesion Detection in CT Scan Images Using the Fuzzy Local Information Cluster Means (FLICM) Automatic Segmentation Algorithm and Back Propagation Network Classification. Asian Pac J Cancer Prev., 18(12): 33953399. doi: 10.22034/APJCP.2017.18.12.3395.

[28] Wang, Y., Jha, S., Chaudhuri, K. (2017). Analyzing the Robustness of Nearest Neighbors to Adversarial Examples.

[29] Kochura, Yu. (2018). Data Augmentation for Semantic Segmentation, 10th Int. Conf. on Advanced Computational Intelligence, Xiamen, China.

[30] Lina, J.M., Mayrand, M. (1995). Complex Daubechies Wavelets, App. Comp. Harmonic Anal., Vol. 2, pp. 219-229.

[31] Burgess, A., Ghandeharian, H. (1984). Visual signal detection. I. Ability to use phase information, J. Opt. Soc. Am., Vol. 1, pp. $900-$ 905.

[32] Chen, S., Beghdadi, A. (2010) Natural enhancement of color image, EURASIP J. Image Video Process., vol. 2010, pp.175203-1175203-19.

[33] Dewangan, S., Sharma, A. (2017). Image Smoothening and Sharpening using Frequency Domain Filtering Technique. International Journal of Emerging Technologies in Engineering Re- search. 5. pp.169-174.

[34] Zhao, H., Shi, J., Qi, J., Wang, X., Jia., J. (2017). Pyramid Scene Parsing Network. Computer Vision and Pattern Recognition (cs.CV). https://arxiv.org/abs/1612.01105. 